\title{
Clinical assessment of magnetic resonance imaging-guided radiofrequency ablation for breast cancer
}

\author{
JIAN LI $^{1 *}$, DAN-DAN WANG ${ }^{3 *}$, YU-NIAN ZHAO ${ }^{1}$, JIAN-WEI ZHOU $^{2}$ and JIN-HAI TANG ${ }^{3}$ \\ ${ }^{1}$ The Affiliated Cancer Hospital of Nanjing Medical University, Jiangsu Cancer Hospital, Jiangsu Institute of Cancer Research, \\ Nanjing, Jiangsu 210000; ${ }^{2}$ Department of Molecular Cell Biology and Toxicology, School of Public Health, Nanjing Medical \\ University, Nanjing, Jiangsu 211166; ${ }^{3}$ Department of General Surgery, The First Affiliated Hospital of \\ Nanjing Medical University, Nanjing, Jiangsu 210029, P.R. China
}

Received May 15, 2018; Accepted May 28, 2019

DOI: $10.3892 /$ mco.2019.1905

\begin{abstract}
The aim of the present study was to investigate the efficacy of magnetic resonance imaging (MRI)-guided radiofrequency ablation (RFA) for breast cancer patients who cannot undergo traditional surgery. A total of 10 patients were treated by MRI-guided RFA, of whom 6 had stage IV disease (lung metastasis $n=3$, bone metastasis $n=1$, liver metastasis $n=1$ and mediastinal metastasis $n=1$ ) and the remaining 4 patients, who refused surgery, had stage III disease accompanied by severe underlying conditions. The changes in feasibility, tumor volume, bleeding, local recurrence, metastasis and complications were evaluated after RFA. The patients were followed up at 1, 3, 6 and 12 months, and annually thereafter. A total of 14 RFA sessions were successfully performed (100\%) in the 10 patients, among whom 7 patients underwent a single RFA session, 2 patients underwent two sessions, and 1 patient underwent three sessions. Compared with pre-RFA, the volume of the tumors at 6 months after RFA was markedly decreased. There was no local tumor recurrence or metastasis detected during a mean follow-up period of 19.5 \pm 3.46 months, and no major complications were reported. Therefore, RFA was found to be a minimally invasive and feasible treatment method in the present study, and MRI-guided RFA may be a promising alternative option for breast cancer patients who are unable to tolerate surgery. However, more prospective studies on the applicability of RFA in breast cancer are required.
\end{abstract}

Correspondence to: Dr Jin-Hai Tang, Department of General Surgery, The First Affiliated Hospital of Nanjing Medical University, 300 Guanzhou Road, Nanjing, Jiangsu 210029, P.R. China

E-mail: jhtang@njmu.edu.cn

*Contributed equally

Key words: breast cancer, radiofrequency ablation, magnetic resonance imaging

\section{Introduction}

Breast cancer is one of the leading cause of cancer-related deaths in women worldwide (1). Surgery is the mainstay of treatment for breast cancer; however, a large number of patients are unable to undergo surgery due to advanced cancer or severe underlying conditions, such as malignant hypertension, diabetes mellitus, poor heart and/or lung function. Therefore, new treatment strategies are urgently required for breast cancer patients with a high surgical risk. Over the past years, several such methods had been investigated, including high-intensity focused ultrasound, laser ablation, microwave ablation and radiofrequency ablation (RFA) (2-5).

RFA is an established procedure that has been widely used in various types of cancer, such as bone metastases, renal cell carcinoma, hepatocellular carcinoma (HCC) and breast cancer (4,6-8). RFA is a local, minimally invasive approach that is accompanied by fewer complications compared with other methods. For example, Lee et al (9) reported that RFA was associated with a better overall and progression-free survival compared with transarterial chemoembolization in single HCC. Chen et al (10), confirmed that computed tomography (CT)-guided RFA was feasible, effective and safe for inoperable pulmonary tumors. A meta-analysis of 15 studies demonstrated that RFA achieved a higher complete ablation rate and a low complication rate in breast cancer, proving that this method is effective and safe (11). However, whether patients diagnosed with advanced breast cancer or synchronous severe underlying conditions can benefit from RFA remains to be investigated.

In the present study, the efficacy of magnetic resonance imaging (MRI)-guided RFA was evaluated in patients with breast cancer at an advanced stage or with a high surgical risk.

\section{Patients and methods}

Patients. The study was conducted at the Department of Breast Surgery of Jiangsu Cancer Hospital and all eligible patients provided written informed consent prior to treatment. Between January 2015 and December 2015, 10 patients were diagnosed by coarse-needle puncture and were all confirmed as invasive breast cancer. All patients were assessed based on MRI, CT 
and related data. Patients with bone metastases were evaluated by emission CT (ECT). The inclusion criteria for RFA were as follows: i) Age >18 years; ii) diagnosis confirmed by pathology; iii) tumor size $\leq 3 \mathrm{~cm}$; iv) tumor distance from the chest wall $\geq 1 \mathrm{~cm}$; v) TNM stage IV; vi) TNM stage III with severe underlying disease; and vii) tumor refractory to various treatments and patient refusing to undergo surgery, chemotherapy and radiotherapy. The exclusion criteria included tumor size $>3 \mathrm{~cm}$, distance from the chest wall $<1 \mathrm{~cm}$, multifocal lesions and good outcome predicted with systemic chemotherapy or radiotherapy. Prior to the initiation of this study, all patients provided written informed consent and the study protocol was approved by the Ethics Committee of Jiangsu Cancer Hospital and Nanjing Medical University.

RFA procedures. Two professional radiologists performed the RFA procedures. All patients underwent routine blood tests, coagulation function and liver function assessment prior to RFA. Tumor stage was assessed by B-mode ultrasonography, CT and MRI. RFA was performed using MedSphere S500 (Medsphere, Carslbad, CA, USA) under MRI guidance (Philips Healthcare, Amsterdam, The Netherlands) and a 17-Ga insulated magnetic-free needle (BD Biosciences, Franklin Lakes, NJ, USA) with an ablation range of 2.5-3.0 cm.

Local anesthesia and disinfection were performed prior to RFA and the needle was inserted into the tumor. Then, MRI scanning at the cross-section, coronal and sagittal planes was performed to obtain three-dimensional stereoscopic images, which confirmed that the needlepoint was at least $0.5-1.0 \mathrm{~cm}$ inside the tumor. At the same time, the volume of tumor was calculated by MRI. Additionally, the power of RFA was gradually adjusted to $50 \mathrm{~W}$ and the temperature of the needle tip was maintained at $100^{\circ} \mathrm{C}$ over $5-10 \mathrm{~min}$. During the process, $0.9 \% \mathrm{NaCl}_{2}$ with $0-5^{\circ} \mathrm{C}$ was circulated into the system continuously to ensure uniform energy distribution. After ablation, the tumor size and blood flow were accessed by MRI. If there was residual tumor, an additional RFA session was immediately performed. After RFA, the patients were observed for $1 \mathrm{~h}$ and any complications were recorded.

Follow-up and outcome measurements. After RFA, all patients were followed up at 1, 3, 6 and 12 months, and annually thereafter: i) The size was assessed by physical examination (palpation); ii) the size, bleeding and apparent diffusion coefficient (ADC) value of the tumor were assessed by MRI; and iii) the size, bleeding and calcification of the tumor were assessed by breast X-ray. Complete remission (CR) was defined as complete disappearance of the tumor and no enhancement on MRI enhanced scan. Partial remission (PR) was defined as a decrease in the tumor volume of $>50 \%$ and non-enhanced area of $>50 \%$ on MRI. Stable disease (SD) was defined as a decrease in the tumor volume of $<50 \%$ or an increase of $<25 \%$, and a non-enhanced area $<50 \%$. Progressive disease (PD) was defined as an increase in the volume of the tumor of $>25 \%$.

Ethics. The procedures followed were in accordance with the ethical standards of the responsible committee on human experimentation (institutional or regional) and with the Helsinki Declaration of 1975, as revised in 2000 (https://www. wma.net/what-we-do/medical-ethics/declaration-of-helsinki/).

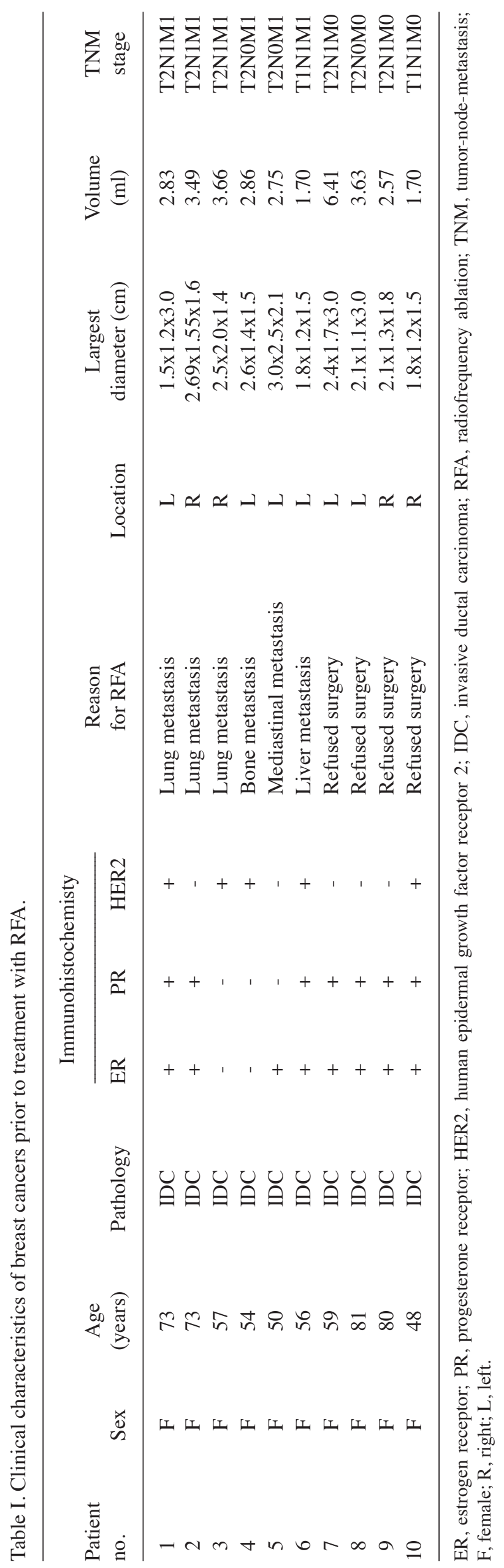



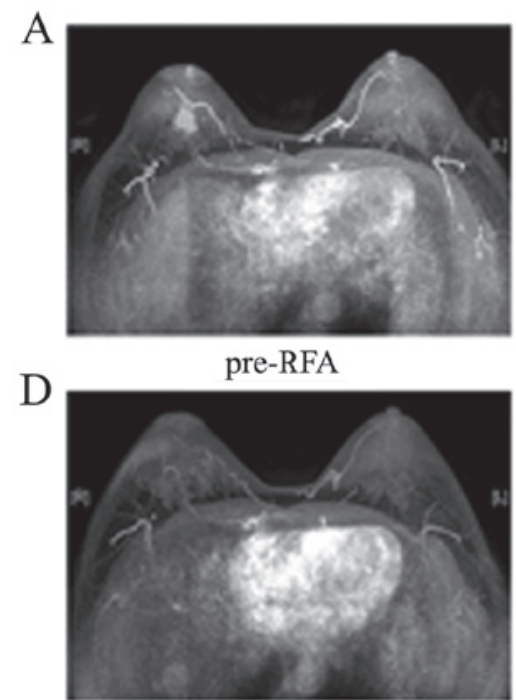

3 months

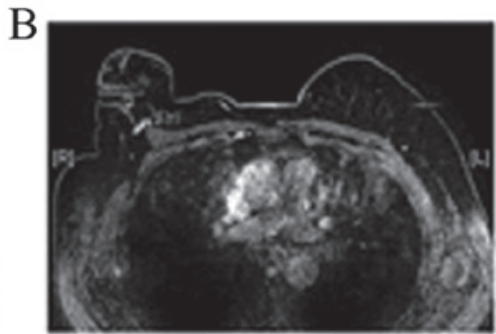

RFA

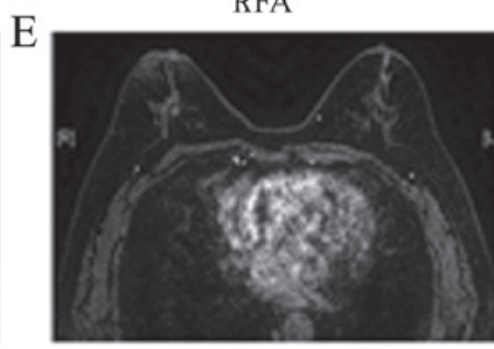

6 months

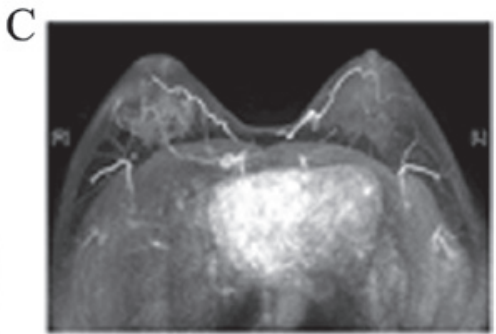

post-RFA

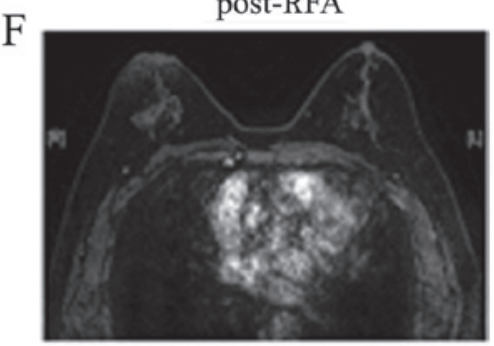

12 months

Figure 1. MRI scans of a patient (A) before, (B) immediately after and at (C) 1, (D) 3, (E) 6 and (F) 12 months after RFA. RFA, radiofrequency ablation; MRI, magnetic resonance imaging.

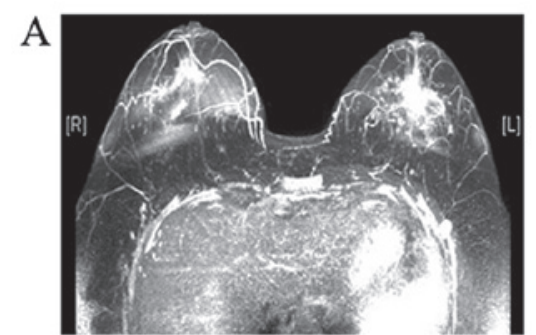

pre-RFA

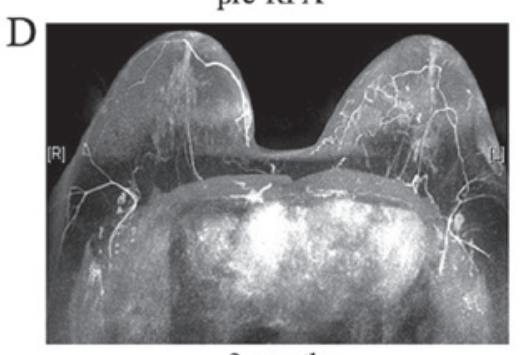

3 months

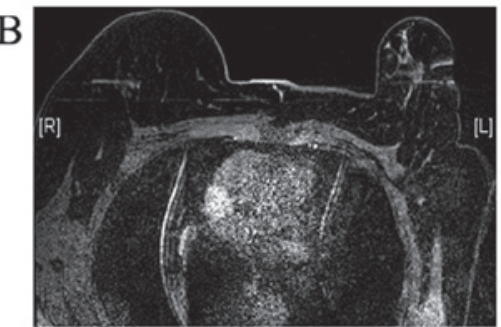

RFA

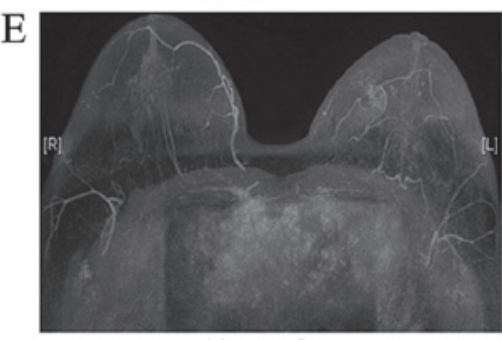

12 months

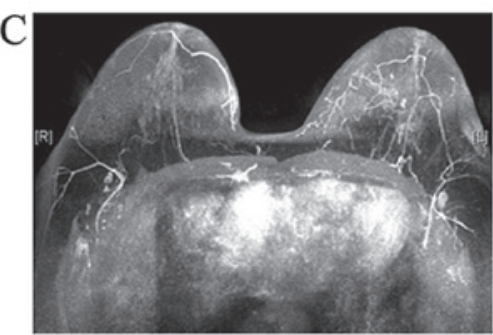

post-RFA

Figure 2. MRI scans of a patient (A) before, (B) immediately after and at (C) 1, (D) 3 and (E) 12 months after RFA. RFA, radiofrequency ablation; MRI, magnetic resonance imaging.

Statistical analysis. Statistical analysis was performed with SPSS 21.0 software (SPSS Inc., Chicago, IL, USA). The pre-/post-treatment volumes were calculated as $\mathrm{V}=\pi \mathrm{abc} / 6$ (V, volume; a, largest diameter; and b and c, the two other perpendicular diameters). The data are presented as mean \pm standard error of the mean. Chi-squared and Fisher's exact tests were used to assess the significance of each clinicopathological factor. Student's t-test was used to evaluate the differences in tumor volume, bleeding and ADC value. $\mathrm{P}<0.05$ was considered to indicate statistically significant differences.

\section{Results}

Patient and clinicopathological characteristics. A total of 10 breast cancer patients who were unable to undergo conventional surgery presented to the Jiangsu Cancer Hospital between January and December 2015. The clinical information of the 10 patients is summarized in Table I. All the patients were women, with a median age of 56 years (range, 48-83 years). A total of 6 patients had stage IV disease (lung metastasis $n=3$, bone metastasis $n=1$, liver metastasis $n=1$ and mediastinal metastasis $n=1$ ) and the remaining 4 patients had stage III disease accompanied by severe underlying conditions.

Outcomes following RFA. A total of 14 RFA sessions were performed in 10 patients, among whom 7 patients underwent a single RFA session, 2 patients received two sessions, and 1 patient received three sessions. Successful placement of the RFA probe was achieved in all patients (100\%). After RFA, all the tumors became necrotic, the blood supply disappeared and no enhancement was observed on MRI (Figs. 1 and 2). 
Table II. Tumor volume pre- and post-RFA.

\begin{tabular}{|c|c|c|c|c|}
\hline Patient no. & Pre-RFA (ml) & 1 month post-RFA (ml) & 6 months post-RFA (ml) & Volume reduction ratio (\%) \\
\hline 1 & 2.83 & 2.80 & 0.22 & 92.2 \\
\hline 2 & 3.49 & 3.01 & 0.28 & 92.0 \\
\hline 3 & 3.66 & 2.81 & 0.11 & 97.0 \\
\hline 4 & 2.86 & 2.00 & 0.00 & 100.0 \\
\hline 5 & 2.75 & 1.85 & 0.00 & 100.0 \\
\hline 6 & 1.70 & 2.20 & 0.00 & 100.0 \\
\hline 7 & 6.41 & 4.12 & 0.45 & 93.0 \\
\hline 8 & 3.63 & 3.50 & 0.24 & 93.4 \\
\hline 9 & 2.57 & 2.23 & 0.13 & 95.0 \\
\hline 10 & 1.70 & 2.44 & 0.00 & 100.0 \\
\hline P-value & & 0.12 & $<0.01$ & \\
\hline
\end{tabular}

RFA, radiofrequency ablation. Bold print indicates statistical significance.

Compared with pre-RFA, the volume of the tumors at 6 months post-RFA had markedly decreased (Table II). No local tumor recurrence or metastasis were detected during a mean follow-up of 19.5 \pm 3.46 months (range, 15-25 months).

Complications. All processes were successfully completed, without any procedure-related or severe complications. The RFA-related complications included one case of pain in the breast and one of perspiration, which were resolved with conservative management.

\section{Discussion}

Although combined-modality therapy has decreased the mortality of breast cancer, a number of patients are unable to undergo surgery due to the high surgical risk associated with old age, advanced stage and severe underlying diseases, or refusal to receive surgery, leading to cancer progression (1). Therefore, different therapeutic strategies are urgently needed. With the advances in technological innovations, new minimally invasive therapeutic alternatives for various solid tumors have been developed, particularly RFA $(10,12,13)$. A systematic review of the relevant literature demonstrated that RFA is a feasible, safe and successful approach to breast cancer treatment, and is associated with only minor complications $(14,15)$.

The present study confirmed the efficacy of MRI-guided RFA in breast cancers with a higher surgical risk. The RFA procedure was safe and all patients tolerated it well, without major complications. In terms of efficacy, RFA reduced the size of the tumor and prevented bleeding. Thus, RFA appears to be a viable treatment option for patients with breast cancer who are not surgical candidates. In addition, it has also been demonstrated that RFA may be used with ultrasound and CT (16). A number of studies have confirmed MRI as the gold standard for assessing the response of breast cancer to RFA treatment (17-19); those studies reported that RFA may be performed in early-stage breast tumors (size range, $0.5-2.0 \mathrm{~cm}$ ); however, the present study indicated that MRI-guided RFA may be an alternative treatment option for breast cancer patients who are not considered candidates for surgery. In addition, accumulating evidence indicates that the most common complications of RFA include pain, skin burns, fever, bleeding and infection, without serious RFA-related complications $(11,20)$. Apart from breast cancer, benign breast diseases may also be treated by RFA. Li et al observed that patients with breast fibroadenoma exhibited rapid recovery, less extensive injury and shorter hospitalization after RFA (21).

Moreover, Wang et al demonstrated that RFA combined with transcatheter arterial chemoembolization prolonged the progression-free survival, median survival time and survival rate of breast cancer patients with liver metastasis (22). In a 4T1 breast cancer animal model, Chiu et al demonstrated that RFA combined with glycated chitosan controlled tumor progression through inducing potent antitumor cytokine responses (23). According to those studies, it may be hypothesized that RFA combined with various other therapies may be applied as rescue treatment for advanced cancers preoperatively or postoperatively, although more evidence-based studies on breast cancer are required.

There were certain limitations to the present study, such as the small number of patients and the fact that it was conducted at a single center. Further multicenter studies with a larger patient sample population are necessary to confirm our results.

In summary, RFA appears to be feasible, effective and safe for breast cancer patients who have surgical contraindications or refuse surgery. Additionally, the value of MRI-guided RFA combined with surgery, chemotherapy, radiotherapy and other treatments warrants further investigation.

\section{Acknowledgements}

Not applicable.

\section{Funding}

The present study was supported by grants from the National Key Research and Development Program of China (no. 2016YFC0905900), the '333' Talent Project of Jiangsu Province [no. 4(2016)], the National Key Clinical 
Specialist Construction Programs of China [no. 544 (2013)] and the Natural Science Foundation of Jiangsu Province (no. BK20151579).

\section{Availability of data and materials}

The datasets generated and analyzed during the present study are available from the corresponding author on reasonable request.

\section{Authors' contributions}

LJ, WD and ZY were responsible for RFA operation. TJ and $\mathrm{ZJ}$ designed the current study and performed statistical analysis. All authors read and approved the final version of this manuscript.

\section{Ethics approval and consent to participate}

All patients provided written informed consent prior to enrolment. The study protocol was approved by the Ethics Committee of Jiangsu Cancer Hospital and Nanjing Medical University. The procedures followed were in accordance with the ethical standards of the responsible committee on human experimentation (institutional or regional) and with the Helsinki Declaration of 1975, as revised in 2000 (http://www. wma.net/e/policy/17-c_e.html).

\section{Patient consent for publication}

All patients approved the publication of the current study.

\section{Competing interests}

The authors declare that they have no competing interests.

\section{References}

1. Siegel RL, Miller KD and Jemal A: Cancer statistics, 2018. CA Cancer J Clin 68: 7-30, 2018.

2. Niederkorn A, Sadoghi B and Komericki P: Pulsed-dye laser therapy for carcinoma in situ of the penis. Br J Dermatol 179: 195-196, 2018.

3. Lunardi A, Cervelli R, Volterrani D, Vitali S, Lombardo C, Lorenzoni G, Crocetti L, Bargellini I, Campani D, Pollina LE, et al: Feasibility of percutaneous intrahepatic split by microwave ablation (PISA) after portal vein embolization for hypertrophy of future liver remnant: The radiological stage-1 ALPPS. Cardiovasc Intervent Radiol 41: 789-798, 2018.

4. Wiksell H, Lofgren L, Schassburger KU, Grundstrom H, Janicijevic M, Lagerstedt U, Leifland K, Nybom R, Rotstein S, Saracco A, et al: Feasibility study on the treatment of small breast carcinoma using percutaneous US-guided preferential radiofrequency ablation (PRFA). Breast 19: 219-225, 2010.

5. Haar GT and Coussios C: High intensity focused ultrasound: Physical principles and devices. Int J Hyperthermia 23: 89-104, 2007.

6. Gazis AN, Beuing O, Franke J, Jollenbeck B and Skalej M: Bipolar radiofrequency ablation of spinal tumors: Predictability, safety and outcome. Spine J 14: 604-608, 2014.
7. Wah TM, Sourbron S, Wilson DJ, Magee D, Gregory WM, Selby PJ and Buckley DL: Renal cell carcinoma perfusion before and after radiofrequency ablation measured with dynamic contrast enhanced MRI: A pilot study. Diagnostics (Basel) 8: E3, 2018.

8. Rajyaguru DJ, Borgert AJ, Smith AL, Thomes RM, Conway PD, Halfdanarson TR, Truty MJ, Kurup AN and Go RS: Radiofrequency ablation versus stereotactic body radiotherapy for localized hepatocellular carcinoma in nonsurgically managed patients: Analysis of the national cancer database. J Clin Oncol 36: 600-608, 2018.

9. Lee SH, Jin YJ and Lee JW: Survival benefit of radiofrequency ablation for solitary $(3-5 \mathrm{~cm})$ hepatocellular carcinoma: An analysis for nationwide cancer registry. Medicine (Baltimore) 96: e8486, 2017.

10. Chen T, Jin J and Chen S: Clinical assessment of computed tomography guided radiofrequency ablation in the treatment of inoperable patients with pulmonary tumors. J Thoracic Dis 9: 5131-5142, 2017.

11. Chen J, Zhang C, Li F, Xu L, Zhu H, Wang S, Liu X, Zha X, Ding Q, Ling L, et al: A meta-analysis of clinical trials assessing the effect of radiofrequency ablation for breast cancer. OncoTargets Ther 9: 1759-1766, 2016

12. Chen K, Zhan MX, Hu BS, Li Y, He X, Fu SR, Xin YJ and Lu LG: Combination of the neutrophil to lymphocyte ratio and the platelet to lymphocyte ratio as a useful predictor for recurrence following radiofrequency ablation of hepatocellular carcinoma. Oncol Lett 15: 315-323, 2018.

13. Bale R, Richter M, Dunser M, Levy E, Buchberger W and Schullian P: Stereotactic radiofrequency ablation for breast cancer liver metastases. J Vasc Interv Radiol 29: 262-267, 2017.

14. Mauri G, Sconfienza LM, Pescatori LC, Fedeli MP, Ali M, Di Leo G and Sardanelli F: Technical success, technique efficacy and complications of minimally-invasive imaging-guided percutaneous ablation procedures of breast cancer: A systematic review and meta-analysis. Eur Radiol 27: 3199-3210, 2017.

15. Nguyen T, Hattery E and Khatri VP: Radiofrequency ablation and breast cancer: A review. Gland Surg 3: 128-135, 2014.

16. Shah DR, Green S, Elliot A, McGahan JP and Khatri VP: Current oncologic applications of radiofrequency ablation therapies. World J Gastrointest Oncol 5: 71-80, 2013.

17. Oura S, Tamaki T, Hirai I, Yoshimasu T, Ohta F, Nakamura R and Okamura Y: Radiofrequency ablation therapy in patients with breast cancers two centimeters or less in size. Breast Cancer 14: 48-54, 2007.

18. Earashi M, Noguchi M, Motoyoshi A and Fujii H: Radiofrequency ablation therapy for small breast cancer followed by immediate surgical resection or delayed mammotome excision. Breast Cancer 14: 39-47, 2007.

19. Yamamoto N, Fujimoto H, Nakamura R, Arai M, Yoshii A, Kaji S and Itami M: Pilot study of radiofrequency ablation therapy without surgical excision for T1 breast cancer: Evaluation with MRI and vacuum-assisted core needle biopsy and safety management. Breast Cancer 18: 3-9, 2011.

20. Hua YQ, Wang P, Zhu XY, Shen YH, Wang K, Shi WD, Lin JH, Meng ZQ, Chen $\mathrm{Z}$ and Chen H: Radiofrequency ablation for hepatic oligometastatic pancreatic cancer: An analysis of safety and efficacy. Pancreatology 17: 967-973, 2017.

21. Li P, Xiao-Yin T, Cui D, Chi JC, Wang Z, Wang T, Qi XX and Zhai B: Evaluation of the safety and efficacy of percutaneous radiofrequency ablation for treating multiple breast fibroadenoma. J Cancer Res Ther 12 (Suppl): C138-C142, 2016.

22. Wang H, Liu B, Long H, Zhang F, Wang S and Li F: Clinical study of radiofrequency ablation combined with TACE in the treatment of breast cancer with liver metastasis. Oncol Lett 14: 2699-2702, 2017.

23. Chiu HY, Leu JD, Chang CY, Lee YJ and Chen WR: Combination of radiofrequency ablation and glycated chitosan as treatment on a syngeneic breast tumor model. Anticancer Res 37: 2965-2974, 2017. 